\title{
An Improved BESO Method for Topology Optimization Design
}

\author{
Mingtao Cui ${ }^{1, a, *}$, Jingling Zhou ${ }^{1, b}$, Xinfeng Yang ${ }^{1, c}$, Yifei Zhang ${ }^{1, d}$ \\ ${ }^{1}$ School of Mechano-electronic Engineering, Xidian University, Xi'an, 710071, China \\ a cuimt@mail.xidian.edu.cn, ${ }^{\mathrm{b}}$ zjlabc88@126.com, ${ }^{\mathrm{c}}$ 1741849580@qq.com, ${ }^{\mathrm{d}}$ 421780789@qq.com \\ *Corresponding author
}

Keywords: Topology optimization, Improved BESO method, Homogenization theory, Periodic boundary condition, Hashin-Shtrikman boundary condition.

\begin{abstract}
Topology optimization design is a technical process that determines the optimal layout of materials with extreme value of objective function. In this paper, an improved bidirectional evolutionary structural optimization (BESO) method for topology optimization design with multiple boundary conditions is proposed. The improved BESO method based on the homogenization theory is utilized to establish the mathematical model of the topology optimization design of microstructure, and the periodic boundary condition and Hashin-Shtrikman boundary condition are taken as boundary constraints. In this method, the advantages of the BESO method are combined with those of the homogenization method. Therefore, this method is suitable for the topology optimization design of microstructure. In addition, reasonable results can be obtained with a single volume constraint by this method. Finally, the effectiveness and feasibility of the proposed method are demonstrated by several typical numerical examples.
\end{abstract}

\section{Introduction}

Structural optimization (including size optimization, shape optimization and topology optimization) in engineering design has been studied for a few decades. By now, it has been largely facilitated and enabled to practice due to the advances in high-performance computing. Topology optimization is one of the material constitutive optimization methods. Topology optimization allows for greater design freedom than size optimization and shape optimization, which deal with variables such as thicknesses, cross-sectional areas of structural members and geometric features of predefined structural configurations.

There are several methods for topology optimization design. The homogenization method, an early and popular method for topology optimization, was first proposed by Bendsøe and Kikuchi [1] This method is represented by a microstructure that redistributes the voids and the materials. Another important method is also originally introduced by Bendsøe [2], and it is referred to as SIMP (solid isotropic microstructure with penalization) method or the power law method (Rozvany et al. [3]). The SIMP method has been recognized as a computationally efficient method for structural topology optimization problems (Rozvany [4]). In the SIMP method, it assumes that the 
material property of all elements is constant, and the design variables are the densities of each element. In fact, SIMP method is a special case of the homogenization method. Another popular method for topology optimization design is level-set-based method [5-8]. The level-set functions [9] are applied to parametric material domain in the methods. The structural boundary is represented exactly where the level-set function is zero. The solid material is represented where the level-set function is positive, and the void is represented where the level-set function is negative. Moreover, evolutionary structural optimization (ESO) proposed by Xie and Steven [10] is also popular for topology optimization design. The problem of single-material topology optimization can be directly solved by ESO method (Bendsøe and Sigmund [11]). In ESO method, the topology optimization of a structure is achieved through removing the elements of material which are the lowest sensitivity of stress energy or strain energy (Xie and Steven [10]; Hinton and Sienz [12]; Querin et al. [13]). However, ESO method only removes elements, not adds elements. Bi-directional evolutionary structural optimization (BESO) method is introduced by Querin et al. [13, 14]. BESO method is an extension of ESO method which allows new elements to be added in the process of topology optimization. Due to the easy implementation, BESO/ESO method has been widely applied in engineering (for example several designs of landmark building [15]). Recently, Xia and Breitkopf [16] proposed a method of material design using topology optimization and energy-based homogenization theory, and solved the problem of topology optimization with single periodic boundary condition.

Combined with the energy-based homogenization theory and BESO theory which are both based on variable density method, an improved BESO method is proposed for microstructure topology optimization design with multiple boundary conditions. In this method, an average sensitivity filtering method is introduced, and a volume rate of evolution is applied to determine the structural volume, which enhances the numerical stability and the convergence rate. Moreover, the optimal microstructure with the periodic boundary conditions and the Hashin-Shtrikman boundary condition can be obtained.

The remaining part of this paper is organized as follows: the next section introduces the energybased homogenization theory. In section 3, the periodic boundary conditions and the HashinShtrikman boundary condition are described. In section 4, the mathematical model of improved BESO method for topology optimization design is established. In section 5, several typical numerical examples are presented to demonstrate the effectiveness of this method. Finally, a conclusion is given in section 6 .

\section{Energy-Based Homogenization Method}

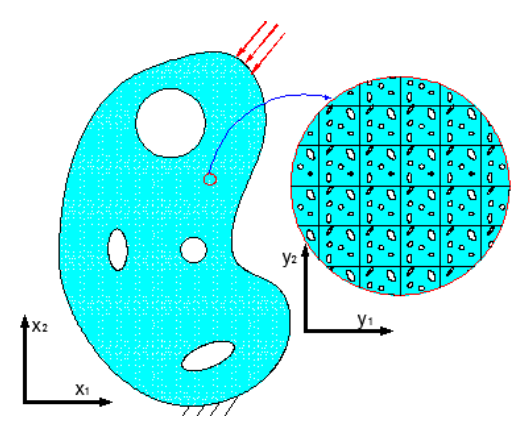

Figure 1 The relationship between the macrostructure (left) and microstructure (right) by a material point constituted.

Homogenization method is an effective topology optimization method for the microstructure of single material. In the scope of linear elasticity, the microstructures of the material are periodic 
distribution in the corresponding macrostructure. The scale relationship between the macrostructure and microstructure is described in the Figure 1, and the relationship between the macro size $x$ and the micro size $y$ can be expressed as

$$
\gamma=\frac{x}{y}
$$

In terms of the asymptotic homogenization, the macro displacement field $u^{\gamma}(x)$ is expanded as

$$
u^{\gamma}(x)=u_{0}(x, y)+\gamma u_{1}(x, y)+\gamma^{2} u_{2}(x, y)+\cdots
$$

When only the first order terms of the above equation is considered, the homogenized stiffness tensor $E_{i j k l}^{H}$ can be written as

$$
E_{i j k l}^{H}=\frac{1}{|Y|} \int_{Y} E_{i j p q}\left(\varepsilon_{p q}^{o(k l)}-\varepsilon_{p q}^{*(k l)}\right) d Y
$$

where $E_{i j k l}^{H}$ is homogenized elasticity tensor by Einstein notation; $E_{i j p q}$ is elasticity tensor by Einstein notation; $\varepsilon_{p q}^{o(k l)}$ is unit test strain fields; and $\varepsilon_{p q}^{*(k l)}$ is periodic fluctuation strain fields.

This paper adopts the energy-based method instead of the asymptotic method. This method imposes the unit strains directly on the boundaries of the base cell. Therefore, the formula $\left(\varepsilon_{p q}^{O(k l)}-\varepsilon_{p q}^{*(k l)}\right)$ corresponds to $\varepsilon_{p q}^{A(k l)}$. In terms of element mutual energies, the base cell $\mathrm{Y}$ is discretized into $N$ finite elements for FEA. Then, Equation (3) is approximately written as

$$
E_{i j k l}^{H}=\frac{1}{|Y|} \sum_{e=1}^{N}\left(\mathbf{u}_{e}^{A(i j)}\right)^{T} \mathbf{k}_{e} \mathbf{u}_{e}^{A(k l)}
$$

where $u_{e}^{A(k l)}$ and $u_{e}^{A(i j)}$ are the element displacement solutions corresponding to $\varepsilon_{p q}^{o(k l)}$, and $\mathbf{k}_{e}$ is the element stiffness matrix. In two-dimension case, the subscript of $E_{i j k l}^{H}$ can be simplified as

$$
\left[\begin{array}{lll}
E_{11}^{H} & E_{12}^{H} & E_{13}^{H} \\
E_{21}^{H} & E_{22}^{H} & E_{23}^{H} \\
E_{31}^{H} & E_{32}^{H} & E_{33}^{H}
\end{array}\right]=\left[\begin{array}{lll}
Q_{11} & Q_{12} & Q_{13} \\
Q_{21} & Q_{22} & Q_{23} \\
Q_{31} & Q_{32} & Q_{33}
\end{array}\right]
$$

where the terms $Q_{i j}$ and element mutual energies $q_{e}^{i j}$ are described as follows:

$$
Q_{i j}=\frac{1}{|Y|} \sum_{e=1}^{N} q_{e}^{i j}, q_{e}^{i j}=\left(\mathbf{u}_{e}^{A(i)}\right)^{T} \mathbf{k}_{e} \mathbf{u}_{e}^{A(j)}
$$

\section{Boundary Conditions}

In this paper, the performances of the microstructure with the periodic boundary conditions (PBC) and Hashin-Shtrikman boundary condition are mainly studied. When a single cell is analyzed, periodic boundary conditions must be applied to the cell to ensure compatibility of deformation and correct computation of stress and strain. Hashin-Shtrikman boundary condition is utilized to deal with the parameterized design variables. 


\subsection{Periodic Boundary Conditions (PBC)}

Under the assumption of periodicity [16], the displacement field of the base cell is as follows:

$$
u_{i}=\varepsilon_{i j}^{0} y_{i}+u_{i}^{*}
$$

where $u_{i}^{*}$ is the micro periodic fluctuation field. Due to the $u_{i}^{*}$ can not be determined, the displacement field becomes

$$
\left\{\begin{array}{l}
u_{i}^{k+}=\varepsilon_{i j}^{0} y_{j}^{k+}+u_{i}^{*} \\
u_{i}^{k-}=\varepsilon_{i j}^{0} y_{j}^{k-}+u_{i}^{*}
\end{array}\right.
$$

where $k+$ and $k$-are a pair of two opposite parallel boundary surfaces which are oriented perpendicular to the $k$-th direction ( $k$ signifies the direction of the coordinates, $k=1,2$ ). The parameter $u_{i}^{*}$ can be eliminated by $u_{i}^{k+}-u_{i}^{k-}$, and $\Delta y_{j}^{k}=y_{j}^{k+}-y_{j}^{k-}$ is constant, i.e. if $j=k, \Delta y_{j}^{k}=y_{j}$, otherwise, $\Delta y_{j}^{k}=0$.

\subsection{Hashin-Shtrikman Boundary Condition}

In this paper, the BESO material interpolation method is used to obtain the density of the interpolation. The elastic tensor is changed as followed

$$
C_{i j l d}\left(\rho_{e}\right)=\rho_{e}^{p} C_{i j l d}^{0}
$$

where $C_{i j l d}^{0}$ is the elastic tensor before optimization, $\rho_{e}$ is the element density, $C_{i j l d}$ is the optimized elastic tensor, the parameter $p$ is the penalization factor.

The Hashin-Shtrikman boundary condition for 2D model can be described as follows

$$
0 \leq k \leq \frac{\rho_{e} k^{0} \mu^{0}}{\left(1-\rho_{e}\right) k^{0}+\mu^{0}}, 0 \leq \mu \leq \frac{\rho_{e} k^{0} \mu^{0}}{\left(1-\rho_{e}\right)\left(k^{0}+2 \mu^{0}\right)+k^{0}}
$$

where $k$ is the bulk modulus, $\mu$ is the shear modulus of elasticity. The parameters $k^{0}$ and $\mu^{0}$ are the bulk modulus and the shear modulus of elasticity of single phase material respectively, and they can be calculated from the following equation

$$
k^{0}=\frac{E^{0}}{2\left(1-v^{0}\right)}, \mu^{0}=\frac{E^{0}}{2\left(1+v^{0}\right)}
$$

where $v^{0}$ is the Poisson ratio. The parameters $(k$ and $\mu)$ are obtained by the following formula

$$
k=\rho_{e}^{p} k^{0}, \mu=\rho_{e}^{p} \mu^{0}
$$

According to the Hashin-Shtrikman boundary condition, the penalization factor $p$ is

$$
p \geq p^{*}\left(v^{0}\right)=\max \left\{\frac{2}{1-v^{0}}, \frac{4}{1+v^{0}}\right\}
$$




\section{Optimization Model based BESO}

\subsection{Problem Statement}

In this paper, an improved method based on the BESO method [17] is applied, so the element elasticity tensor $E_{e}$ is defined as

$$
E_{e}\left(\rho_{e}\right)=E_{\min }+\rho_{e}^{p}\left(E_{0}-E_{\min }\right)
$$

where $E_{0}$ is the elasticity tensor of solid material, and $E_{\min }$ is the elasticity tensor of void material. Here $E_{\min }$ is defined $10^{-9}$ instead of 0 , thus avoiding the singularity.

In this paper, the improved BESO method is used to obtain the density of the interpolation based on the BESO method, and the topology optimization problem is described as

$$
\left\{\begin{array}{l}
\underset{\rho}{\min } c=c\left(E_{i j k l}^{H}(\rho)\right) \\
\text { s.t.: } \mathbf{K} \mathbf{U}^{A(k l)}=\mathbf{F}^{k l}, \quad k, l=1, \ldots, d \\
\text { s.t.: } \sum_{e=1}^{N} \frac{v_{e} \rho_{e}}{|Y|} \leq \alpha \\
\text { s.t. }: \mathbf{K U}=\mathbf{T} \\
\text { s.t. }: 0 \leq \rho_{e} \leq 1, e=1, \ldots, N
\end{array}\right.
$$

where $\mathbf{U}^{A(k l)}$ is the displacement vector, $\mathbf{F}^{k l}$ is the force vector. The parameter $d$ is the spatial dimension, $v_{e}$ is the element volume, and $\alpha$ is the maximum volume fraction. For $2 \mathrm{D}$ case, the objective $c\left(E_{i j k l}^{H}(\rho)\right)$ minimization (the maximization of the material bulk modulus) is

$$
c=-\left(E_{1111}+E_{1122}+E_{2211}+E_{2222}\right)
$$

When the maximization of material shear modulus is considered

$$
c=-E_{1212}
$$

The flowchart of the proposed method to solve the topology optimization problem of microstructure is shown in Figure 2. 


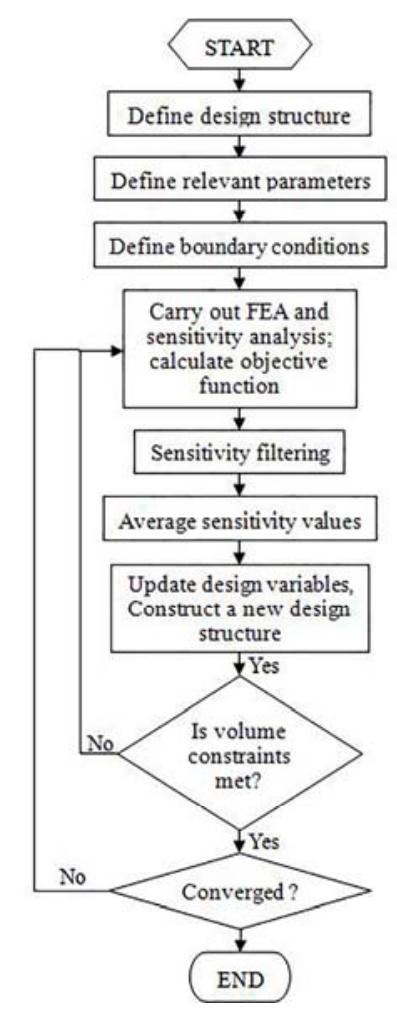

Figure 2 The diagram of the numerical implementation process by the improved BESO method.

\subsection{Sensitivity Analysis based BESO}

The sensitivity of this topology optimization can be described as $\frac{\partial c}{\partial \rho}$. The sensitivity number in BESO that shows the relative ranking of all sensitivities can be defined as:

$$
\alpha_{i}=\frac{1}{p} \frac{\partial c}{\partial \rho_{i}}
$$

where $c$ denotes the bulk modulus or the shear modulus. $\alpha_{i}$ is the sensitivity value of the $i$-th element.

Topology optimization usually goes with numerical instabilities such as checkerboard and meshdependency. To void those phenomena, a mesh-dependency filter is used in this work. Then, the nodal sensitivity numbers are converted back into element before the topology can be determined based on the image-process techniques $[18,19]$. The filter functions are based on a length-scale $r_{\min }$ which is to identify the nodes that influence the sensitivity of the $i$-th element. The sensitivity is modified by sensitivity filter as follows:

$$
\overline{\alpha_{i}}=\frac{\sum_{j=1}^{M} w\left(r_{i j}\right) \alpha_{j}^{n}}{\sum_{j=1}^{M} w\left(r_{i j}\right)}
$$

When the density filter is considered, the densities are transformed into $\overline{\rho_{i}}$ as follows

$$
\overline{\rho_{i}}=\frac{\sum_{j=1}^{M} w\left(r_{i j}\right) \rho_{i}}{\sum_{j=1}^{M} w\left(r_{i j}\right)}
$$


Assume that a circle of radius $r_{\min }$ centred at the centroid of the $i$-th element is drawn. Thus a circular sub-domain $\Omega_{i}$ is generated, and the paramter $M$ is the number of nodes in $\Omega_{i}, r_{i j}$ is the distance between the center of the element $i$ and node $j . \alpha_{j}^{n}$ is the node sensitivity value of the $j$-th element. $w\left(r_{i j}\right)$ is the weight factor which is given as

$$
w\left(r_{i j}\right)=\left\{\begin{array}{lll}
r_{\min }-r_{i j} & \text { for } & r_{i j}<r_{\text {min }} \\
0 & \text { for } & r_{i j} \geq r_{\text {min }}
\end{array}\right.
$$

In the BESO algorithm, the objective function and topology is impossible to be convergent because only two discrete design variables $\rho_{\text {min }}$ and 1 is applied. A method of averaging sensitivity number with its historical information is applied to improve the primary sensitivity number [19]. The modified sensitivity is as follows

$$
\overline{\overline{\alpha_{i}}}=\frac{\bar{\alpha}_{i, T}+\bar{\alpha}_{i, T-1}}{2}
$$

where $T$ denotes the $T$-th iteration. Let $\bar{\alpha}_{i, T}=\overline{\bar{\alpha}}_{i}$, which will be used in the next iteration, thus the sensitivity information is considered in the previous iteration. This method can control the change of design variables for solid and void elements. Therefore, this averaging sensitivity number method can greatly stabilizes the evolution process.

\subsection{Optimality Criteria Method}

The optimization problem (15) can be solved by using many approaches such as Optimality Criteria (OC) method, Method of Moving Asymptotes (MMA) and Sequential Linear Programming (SLP) and so on. This paper will use a standard OC method to solve the optimization problem.

Following the heuristic updating scheme [11], the design variables can be formulated as

$$
\rho_{e}^{\text {new }}=\left\{\begin{array}{lll}
\max \left(0, \rho_{e}-m\right) & \text { for } & \rho_{e} B_{e}^{\eta} \leq \max \left(0, \rho_{e}-m\right) \\
\min \left(1, \rho_{e}+m\right) & \text { for } & \rho_{e} B_{e}^{\eta} \geq \max \left(0, \rho_{e}-m\right) \\
\rho_{e} B_{e}^{\eta} & , & \text { others }
\end{array}\right.
$$

where $m$ is a move limit, which is usually set as $0.2 ; \eta$ is a damping coefficient; and $B_{e}$ can be obtained from the optimality condition as follows:

$$
B_{e}=\frac{-\frac{\partial c}{\partial \rho_{\mathrm{e}}}}{\lambda \frac{\partial V}{\partial \rho_{e}}}
$$

where $\lambda$ is the Lagrangian multiplier that can be selected by the bi-section algorithm to satisfy the constraint of material volume fraction. The structural volume is determined by the iterative formula, the target volume $V^{*}$ and volume rate of evolution er. Therefore, the structural volume is defined as

$$
V_{T+1}=\left\{\begin{array}{lll}
\max \left(V_{T}(1-e r), V^{*}\right) & \text { if } \quad V_{T} \geq V^{*} \\
\min \left(V_{T}(1+e r), V^{*}\right) & \text { if } \quad V_{T}<V^{*}
\end{array}\right.
$$




\subsection{Numerical Solution of the Homogenization Equations}

The global displacement vector $\mathbf{U}$ is separated into four parts: $\overline{\mathbf{U}}_{1}$ is the prescribed displacement values; $\mathbf{U}_{2}$ is the interior nodes displacement; $\mathbf{U}_{3}$ and $\mathbf{U}_{4}$ are the displacements of nodes which located on the boundaries of the base cell, and they satisfy the relationship $\mathbf{U}_{4}=\mathbf{U}_{3}+\overline{\mathbf{W}}$. The parameter $\overline{\mathbf{W}}$ can be calculated by (8). The constraint $\mathbf{K} \mathbf{U}^{\mathbf{A}(k l)}=\mathbf{F}^{k l}$ can be expanded to

$$
\left[\begin{array}{llll}
\mathbf{K}_{11} & \mathbf{K}_{12} & \mathbf{K}_{13} & \mathbf{K}_{14} \\
\mathbf{K}_{21} & \mathbf{K}_{22} & \mathbf{K}_{23} & \mathbf{K}_{24} \\
\mathbf{K}_{31} & \mathbf{K}_{32} & \mathbf{K}_{33} & \mathbf{K}_{34} \\
\mathbf{K}_{41} & \mathbf{K}_{42} & \mathbf{K}_{43} & \mathbf{K}_{44}
\end{array}\right]\left[\begin{array}{l}
\mathbf{U}_{1} \\
\mathbf{U}_{2} \\
\mathbf{U}_{3} \\
\mathbf{U}_{4}
\end{array}\right]=\left[\begin{array}{l}
\mathbf{F}_{1} \\
\mathbf{F}_{2} \\
\mathbf{F}_{3} \\
\mathbf{F}_{4}
\end{array}\right]
$$

where $\mathbf{F}_{1}$ equals to the reaction forces at the nodes with prescribed displacements, $\mathbf{F}_{2}=0$ and $\mathbf{F}_{3}+\mathbf{F}_{4}=0$. Because $\mathbf{K}$ is a symmetric matrix, i.e. $\mathbf{K}_{\mathrm{ij}}=\mathbf{K}_{\mathrm{ji}}$, Formula (26) can be converted into

$$
\begin{aligned}
& {\left[\begin{array}{cc}
\mathbf{K}_{22} & \mathbf{K}_{23}+\mathbf{K}_{24} \\
\mathbf{s y m} . & \mathbf{K}_{33}+\mathbf{K}_{34}+\mathbf{K}_{43}+\mathbf{K}_{44}
\end{array}\right]\left[\begin{array}{c}
\mathbf{U}_{2} \\
\mathbf{U}_{3}
\end{array}\right]} \\
& =-\left[\begin{array}{c}
\mathbf{K}_{21} \\
\mathbf{K}_{31}+\mathbf{K}_{41}
\end{array}\right] \overline{\mathbf{U}_{1}}-\left[\begin{array}{c}
\mathbf{K}_{24} \\
\mathbf{K}_{34}+\mathbf{K}_{44}
\end{array}\right] \overline{\mathbf{W}}
\end{aligned}
$$

\section{Numerical Examples}

\subsection{D Examples for Maximizing the Bulk Modulus}

In this case, a single cell $\mathrm{Y}$ is discretized into $100 \times 100$ 4-node quadrilateral elements. The Young's modulus of the solid material is set as $E_{0}=1$ and the Poisson's ratio is set as $v=0.3$. The maximum volume fraction, the evolution rate, the filter radius and penalty exponent are set as $V^{*}=0.5$, er $=0.02, r=3$ and $p=3$, respectively. Figure 3 describes the different results with different filter methods, where, the blue regions and green regions denote solid material and the void, respectively.

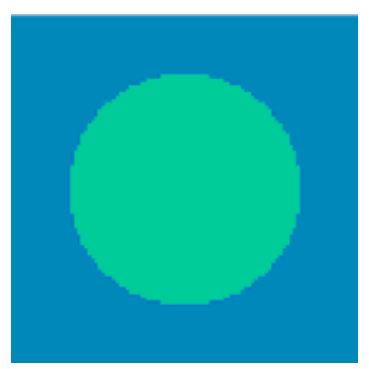

(a)

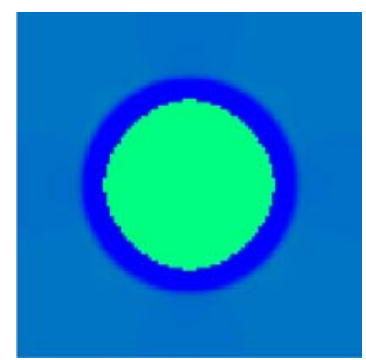

(b)

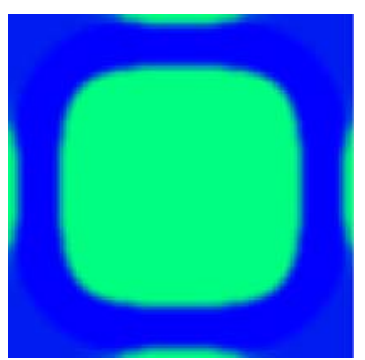

(c)

Figure 3 The optimal solutions of a single cell with different filter methods. ((a) The initial design domain; (b) The optimal microstructure which is obtained by the sensitivity filtering $(c=-0.4776$, iteration: 132); (c) The optimal microstructure which is obtained by the density filtering $(c=-0.6474$, iteration: 256)). 


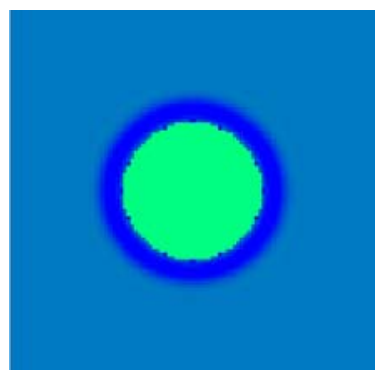

(a)

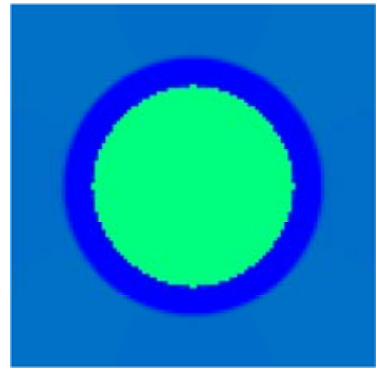

(c)

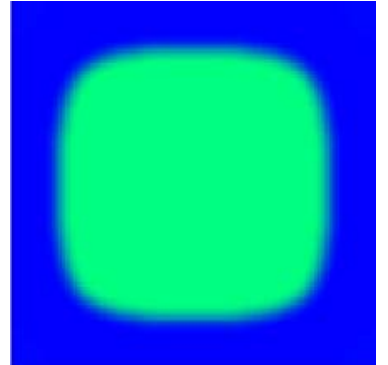

(b)

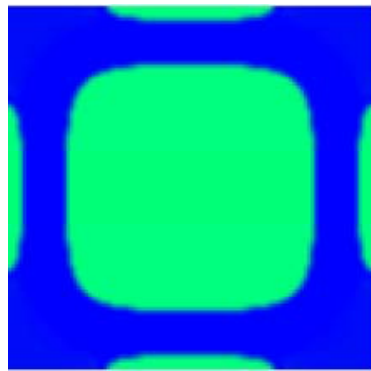

(d)

Figure 4 The optimal solutions of a single cell with penalty factor $p=3$ and different filter radii. ((a) The optimal microstructure which is obtained by the sensitivity filtering and the filter radius $\mathrm{r}=5$ ( $c=-0.4220$,iteration: 225); (b) The optimal microstructure which is obtained by the density filtering and the filter radius $\mathrm{r}=5$ ( $c=-0.6536$,iteration: 241$)$; (c) The optimal microstructure which is obtained by the sensitivity filtering and the filter radius $r=2(c=-0.2596$, iteration: 123$)$; (d) The optimal microstructure which is obtained by the density filtering and the filter radius $\mathrm{r}=2(c=-$ 0.3395, iteration: 455)).

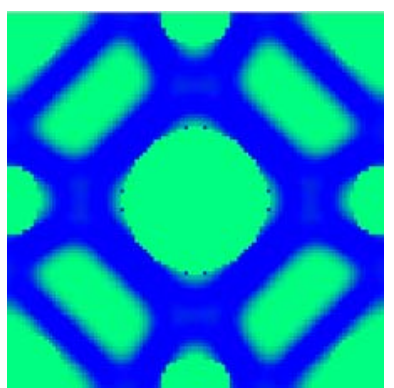

(a)

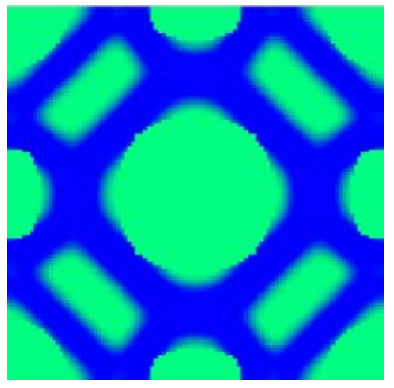

(c)

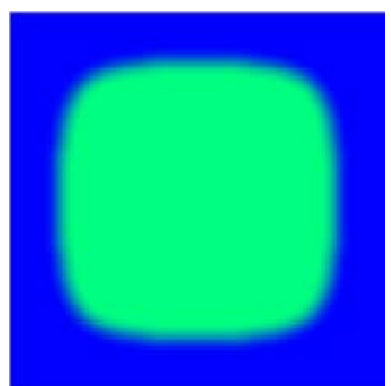

(b)

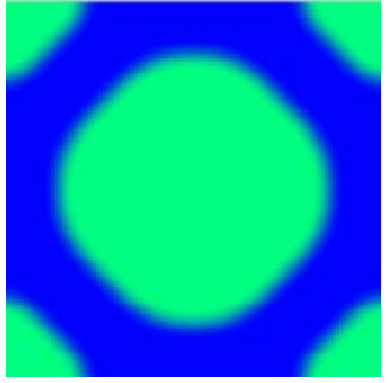

(d)

Figure 5 The optimal solution of a single cell with the filter radius $\mathrm{r}=5$ and different penalty factors. ((a) The optimal microstructure which is obtained by the sensitivity filtering and penalty factor $\mathrm{p}=5$ 
( $c=-0.55816$, iteration: 396); (b) The optimal microstructure which is obtained by the density filtering and the penalty factor $\mathrm{p}=5(c=-0.6206$, iteration:218); (c) The optimal microstructure which is obtained by the sensitivity filtering and penalty factor $p=7(c=-0.5544$, iteration: 396$)$; (d) The optimal microstructure which is obtained by the density filtering and the penalty factor $\mathrm{p}=7$ ( $c=-$ 0.5590, iteration: 51)).

To study the influences of the filter radius and the penalty factor, two cases are proposed in this paper. In case 2 (Figure. 4), all parameters are the same as those in case 1, but the filter radii are defined as $r=5$ and $r=2$, respectively. In case 3 (Figure. 5), all parameters are the same as those in case 2 , but the penalty factors are set as $p=5$ and $p=7$, respectively. The objective function value $c$ and the number of iterations are shown in each figure.

From the above cases, it can be found that the boundaries of optimal microstructures are blurry using the sensitivity filter method. This is because the middle densities are not avoided in the homogenization method. The optimization structure with density filtering has a more clear material layout with density filtering than that with sensitivity filtering, and their objective function values are bigger than the cases with sensitivity filtering. The smaller the filter radius, the clearer the microstructure when the density filtering is applied. From the case3, it can be observed that both sensitivity filtering and density filtering have little influence with the penalty factor value, but the objective function value is little change. Moreover, it can be found that several reasonable optimal results with stability and quickness are obtained by the proposed method.

\subsection{D Examples for Maximizing the Shear Modulus}

In this section, a single cell $\mathrm{Y}$ is discretized into $100 \times 100$ 4-node quadrilateral elements. The Young's modulus of the solid material is $E_{0}=1$ and the Poisson's ratio $v=0.3$. The maximum volume fraction is given $V^{*}=0.5$. The parameter evolution rate is $e r=0.02$. The initial design domain is same to the above cases of the maximizing the bulk modulus (Figure 3(a)). It is worth noting that different optimal results may be obtained with different parameters.

The following figures describe the different optimal results with different filter methods and different parameters. 


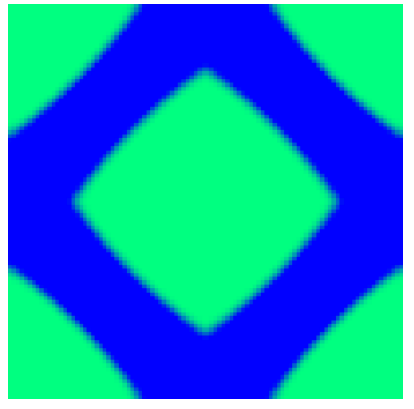

(a)

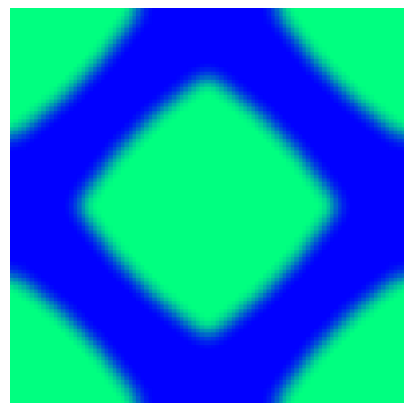

(d)

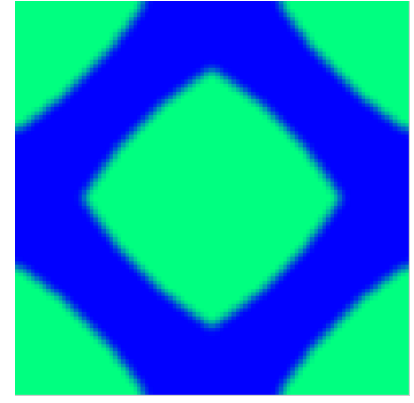

(b)

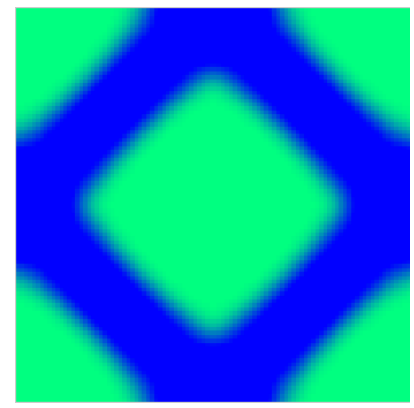

(e)

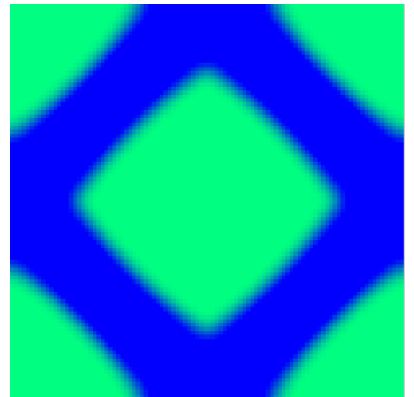

(c)

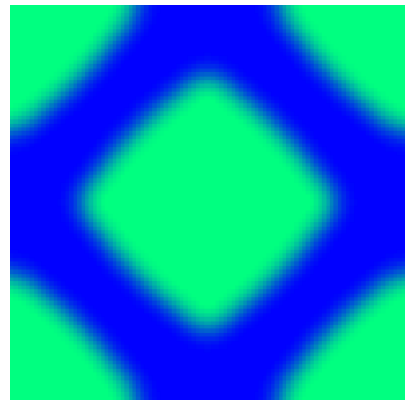

(f)

Figure 6 The optimal results with different parameters ((a) The optimal result with sensitivity filtering ( $p=3, r=3)$ after 33 iterations; (b) The optimal result with density filtering $(p=3, r=3)$ after 50 iterations; (c)The optimal result with sensitivity filtering $(p=3, r=5)$ after 27 iterations; (d) The optimal result with density filtering $(\mathrm{p}=3, \mathrm{r}=5)$ after 77 iterations; (e) The optimal result with sensitivity filtering $(\mathrm{p}=3, \mathrm{r}=7)$ after 22 iterations; (f) The optimal result with density filtering $(\mathrm{p}=3$, $\mathrm{r}=7$ ) after 81 iterations).

Table 1 The changes of objective function $c$ with the change of algorithm parameters.

\begin{tabular}{|c|c|c|c|}
\hline $\begin{array}{c}\text { Penalty Factor } \\
p\end{array}$ & $\begin{array}{c}\text { Filter Radius } \\
r\end{array}$ & $\begin{array}{c}\text { Objective Function } c \\
\text { (Sensitivity Filtering) }\end{array}$ & $\begin{array}{c}\text { Objective Function } c \\
\text { (Density Filtering) }\end{array}$ \\
\hline 3 & 5 & -0.1254 & -0.1214 \\
\hline 3 & 3 & -0.1312 & -0.1284 \\
\hline 3 & 7 & -0.1196 & -0.1144 \\
\hline
\end{tabular}




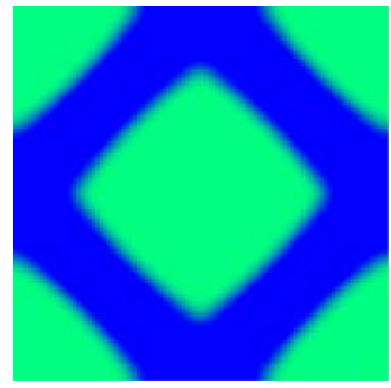

(a)

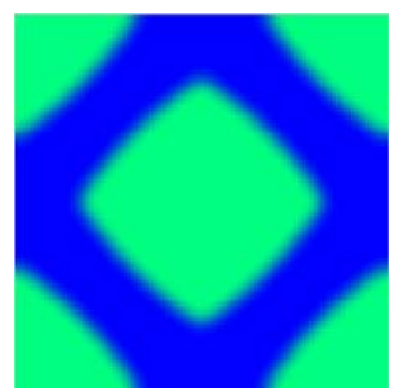

(d)

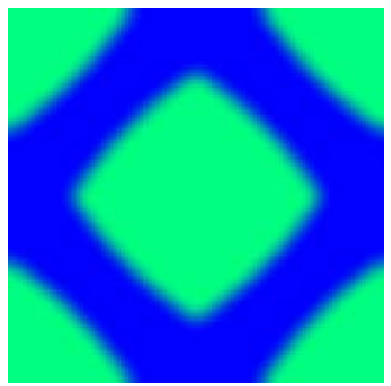

(b)

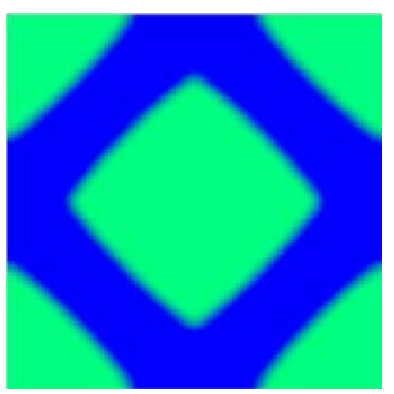

(e)

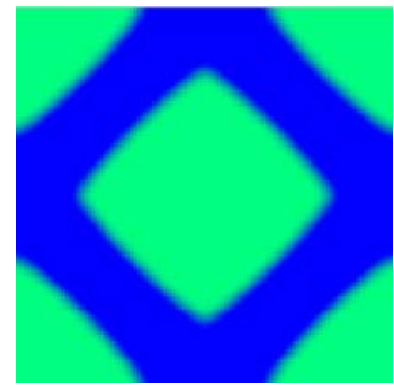

(c)

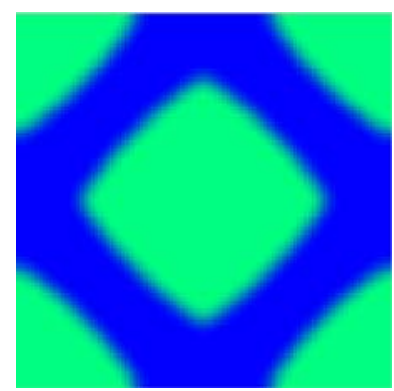

(f)

Figure 7 The optimal results with different parameters ((a) The optimal results with sensitivity filtering ( $p=3, r=5)$ after 27 iterations; (b) The optimal results with density filtering $(p=3, r=5)$ after 77 iterations; (c)The optimal results with sensitivity filtering ( $\mathrm{p}=5, \mathrm{r}=5)$ after 15 iterations; (d) The optimal results with density filtering $(\mathrm{p}=5, \mathrm{r}=5)$ after 65 iterations; (e) The optimal results with sensitivity filtering ( $p=7, r=5)$ after 15 iterations; (f) The optimal results with density filtering $(p=7$, $\mathrm{r}=5$ ) after 51 iterations).

Table 2 The change of objective function $c$ with the change of algorithm parameters.

\begin{tabular}{|c|c|c|c|}
\hline $\begin{array}{c}\text { Penalty Factor } \\
\mathrm{p}\end{array}$ & $\begin{array}{c}\text { Filter Radius } \\
\mathrm{r}\end{array}$ & $\begin{array}{c}\text { Objective c } \\
\text { (Sensitivity Filtering) }\end{array}$ & $\begin{array}{c}\text { Objective c } \\
\text { (Density Filtering) }\end{array}$ \\
\hline 3 & 5 & -0.1254 & -0.1214 \\
\hline 5 & 5 & -0.1232 & -0.1138 \\
\hline 7 & 5 & -0.1222 & -0.1096 \\
\hline
\end{tabular}

From Table 1 and Table 2, it can be observed that both sensitivity filtering method and density filtering method have little influence with different penalty factors and filter radii for the objective function (maximum shear modulus). Therefore, the proposed method is suitable for topology optimization of microstructures, by which reasonable results with good stability can be obtained.

\section{Conclusions}

In this paper, an improved BESO topology optimization method with multiple boundary conditions is proposed. The maximization of the material bulk modulus and the maximization of material shear modulus are taken as the objective of microstructural topology optimization with the volume constraints, respectively. The mathematical model of topology optimization is established by combining the BESO method with the homogenization theory, and it satisfies the periodic boundary condition and Hashin-Shtrikman boundary condition simultaneously. The effectiveness and feasibility of proposed method are demonstrated by several typical numerical examples. The proposed method is suitable for topology optimization of microstructures and reasonable results can 
be obtained with fast convergence. Moreover, the numerical instability can be avoided by this method.

\section{References}

[1] Bendsøe, M.P., Kikuchi, N. (1988) Generating optimal topologies in structural design using a homogenization method. Comput Method Appl Mech Eng, 71(2), 197-224.

[2] Bendsøe, M.P. (1989) Optimal shape design as a material distribution problem. Struct Optim, 1, 193-202.

[3] Rozvany, G.I.N., Zhou, M., Birker, T. (1992) Generalized shape optimization without homogenization. Struct Optim, 4, 250-254.

[4] Rozvany, G.I.N. (2001) Aims, scope, methods, history and unified terminology of computer-aided topology optimization in structural mechanics. Struct Multidisc Optim, 21(2), 90-108.

[5] Sethian, J.A.,Wiegmann, A. (2000) Structural boundary design via level set and immersed interface methods. Journal of Computational Physics, 163(2), 489-528.

[6] Osher, S.J., Santosa, F. (2001) Level set methods for optimization problems involving geometry and constraints I. frequencies of a two-density inhomogeneous drum. Journal of Computational Physics, 171(1), 272288.

[7] Allaire, G., Jouve, F., Toader, A.M. (2004) Structural optimization using sensitivity analysis and a level-set method. Journal of Computational Physics, 194(1), 363-393.

[8] Wang, M.Y., Wang, X., Guo, D. (2003) A level set method for structural topology optimization. Computer Methods in Applied Mechanics and Engineering, 192(1-2), 227-246.

[9] Sethian, J.A. (1999) Level Set Methods and Fast Marching Method:Evolving Interfaces in Computational Geometry, Fluid Mechanics, Computer Vision, and Materials Science. Cambridge University Press: Cambridge.

[10] Xie, Y.M., Steven, G.P. (1993) A simple evolutionary procedure for structural optimization. Comput Struct, 49, 885-896.

[11] Bendsøe, M.P., Sigmund O. (2003) Topology optimization: theory, methods and applications, Springer.

[12] Hinton, E., Sienz, J. (1995) Fully stressed topological design of structures using an evolutionary procedure.

Eng Comput, 12, 229-244.

[13] Querin, O.M., Steven, G.P., Xie, Y.M. (1998) Evolutionary structural optimization (ESO) using a bidirectional algorithm. Eng Comput, 15, 1031-1048.

[14] Querin, O.M., Young, V., Steven, G.P., Xie, Y.M. (2000) Computational efficiency and validation of bidirectional evolutionary structural optimization. Comput Methods Appl Mech Eng, 189, 559-573.

[15] Cui, C., Ohmori, H., Sasaki, M. (2005) Structural design by extended ESO method. Proceedings of frontiers of computational sciences symposium, 149-156.

[16] Xia, L., Breitkopf, P. (2015) Design of materials using topology optimization and energy-based homogenization approach in Matlab. Struct Multidisc Optim, 1, 1-13.

[17] Huang, X.D., Xie, Y.M. (2010) A further review of ESO type methods for topology optimization. Struct Multidisc Optim, 41, 671-683.

[18] Huang, X., Xie, Y.M. (2000) Evolutionary topology optimization of continuum structures: methods and applications. John Wiley \& Sons.

[19] Huang, X., Xie, Y.M. (2009) Bi-directional evolutionary topology optimization of continuum structures with one or multiple materials. Comput Mech, 43(3), 393-401. 